\title{
An Information-Theoretic and Dissipative Systems Approach to the Study of Knowledge Diffusion and Emerging Complexity in Innovation Systems
}

\author{
Guillem Achermann ${ }^{1}\left(\mathbb{D}\right.$, Gabriele De Luca ${ }^{1(\triangle)}(\mathbb{D}$, \\ and Michele Simoni ${ }^{2}$ (D) \\ ${ }^{1}$ RUDN University, Moscow, Russia \\ gabriele.deluca@mail.ru \\ ${ }^{2}$ University of Naples Parthenope, Naples, Italy
}

\begin{abstract}
The paper applies information theory and the theory of dissipative systems to discuss the emergence of complexity in an innovation system, as a result of its adaptation to an uneven distribution of the cognitive distance between its members. By modelling, on one hand, cognitive distance as noise, and, on the other hand, the inefficiencies linked to a bad flow of information as costs, we propose a model of the dynamics by which a horizontal network evolves into a hierarchical network, with some members emerging as intermediaries in the transfer of knowledge between seekers and problem-solvers. Our theoretical model contributes to the understanding of the evolution of an innovation system by explaining how the increased complexity of the system can be thermodynamically justified by purely internal factors. Complementing previous studies, we demonstrate mathematically that the complexity of an innovation system can increase not only to address the complexity of the problems that the system has to solve, but also to improve the performance of the system in transferring the knowledge needed to find a solution.
\end{abstract}

Keywords: Knowledge diffusion - Innovation system - Hierarchical networks · Dissipative systems

\section{A Network and Systems Approach to Problem-Solving}

\subsection{Networks of Innovation as Information and Knowledge Processing Systems}

The analysis of networks through sociometry has encouraged social scientists since the beginning of the twentieth century [1] to calculate degrees of strength or density of connections between different organisations located in a network. At the same time, efforts to understand the innovation process have led researchers to abandon the idea of a linear process of innovation, and to propose instead evolutionary models where the

Authors are listed in alphabetical order. 
formalization and organization of a network becomes strategic to accelerate the flow of information and knowledge and the emergence of innovation [2]. Several forms of reticular organization (hierarchical, heterarchical, according to the centrality of elements, according to the transitivity of element, etc.) can be conceptualized within that context. Evolutionary economics and technology studies highlight (neo-Schumpeterian) models to understand the plurality of evolution cases, depending on the initial forms of organization, but also on the ability of a system to adapt to systemic crises.

In this work we study, from an information-theoretical perspective, the relationship between the structure of an innovation network, the noise in its communication channels and the energy costs associated with the network's maintenance. An innovation network is here considered to encompass a variety of organisations who, through their interactions and the resulting relationships, build a system conducive to the emergence of innovation. This system is identified by the literature [3] with different terms, such as innovation ecosystem, [4] problem-solving network, [5] or innovation environment [6]. In this system, the information channels transfer a multitude of information and knowledge which, depending on the structural holes, $[7,8]$ but also on the absence of predetermined receivers, are subject to information "noise" [9]. The more the information is distorted in the network, the more energy is needed to transfer accurate information, in order to keep performance of the innovation network high. The idea we propose is that the structure of an innovation system evolves to address the heterogeneity in the quality of communication that takes place between its members. In particular, we argue that the noise in a network increases the complexity of the network structure required for the accurate transfer of information and knowledge, and thus leads to the emergence of hierarchical structures. These structures, thanks to their fractal configuration, make it possible to combine high levels of efficiency in the transmission of information, with low network maintenance costs. This idea complements previous studies that have analysed the relationship between the structure of an innovation network, on one hand, and the complexity of the problem to be solved and the resulting innovation process, on the other, [10] by focusing on communication noise and cost of network structure maintenance. To the existing understanding of this phenomenon we contribute by identifying a thermodynamically efficient process which the network follows as it decreases in entropy while simultaneously cutting down its costs.

This model is based on the analysis of a network composed of two classes or categories of organisations, which operate within the same innovation system [11]. These classes are represented by a central organisation called seeker, which poses a research question to a group of other organisations, called problem-solvers, and from which in turn receives a solution. It has been suggested [12] that one of the problems that the innovation system has to solve, and for which it self-organises, is the problem of effective diffusion of knowledge between problem-solvers and solution-seekers, as this can be considered as a problem sui generis. The theory on the diffusion of knowledge in an innovation system suggests that this problem is solved through the evolution of modular structures in the innovation network, which implies the emergence of organisations that act as intermediary conduits of knowledge between hyperspecialised organisations in the same innovation environment [13]. A modular structure is, in network theory, connected to the idea of a hierarchical or fractal structure of the network, [14] and is also characterised by scale-invariance; [15] the latter is a particularly important property, because if innovation systems have it as an emergent 
property of their behaviour, this allows them to be considered as complex adaptive systems [16]. It has been suggested that scale-invariance property of an innovation system might emerge as the result of horizontal cooperation between its elements, [17] which try to reach the level of complexity required to solve a complex problem; but it is not yet clear how does a complex structure emerge when the complexity of the problem does not vary, which is a phenomenon observed empirically $[18,19]$. In this paper we show how complexity can also vary as a result of a non-uniform distribution of the cognitive distance between organisations of the network, and of the adaptation required to solve the problem of knowledge diffusion among them. Our contribution to the theoretical understanding on the self-organising properties of innovation systems is that, by framing the problem of heterogeneous cognitive distance between organisations under the theory of dissipative systems, we can explain in thermodynamically efficient terms the reduction in entropy of an innovation system, as an emergent adaptation aimed at reducing costs of maintenance of the system's structure.

\subsection{Self-organisation and Complexity in Dissipative Innovation Systems}

The theoretical framework which we use for this paper is comprised by four parts. First, we will frame the innovation system as a thermodynamically-open system, which is a property that derives from the fact that social systems also are [20]. Second, we will see under what conditions a system can undertake self-organisation and evolution. This will allow us to consider an innovation system as a complex adaptive system, should it be found that there are emergent properties of its behaviour which lead to an increase in complexity. Third, we will frame the innovation system as a dissipative system, which is a property also shared by social systems [21]. Dissipative systems are characterised by the fact that a variation in the level of their entropy tends to happen as a consequence of their changed ability to process inputs, and we will see how this applies for innovation systems. Lastly, we will study cognitive distance as it applies to a network of innovation, in order to show how a spontaneous reduction in it leads to an increase in complexity of the network.

Thermodynamically-Open Innovation Systems. An open thermodynamic system is defined as a system which exchanges matter and energy with its surrounding environment, [22] and among them are found all social systems, which are open systems due to their exchanging of energy with the surrounding environment [23]. Social systems are also dynamical systems, because their structure changes over time through a process of dynamical evolution [24]. Innovation systems are some special classes of social systems, [25] which can thus also be considered as open systems [26]. In addition to this, like all social systems, innovation systems are also capable of selforganisation, [27] which is a property that they inherit from social systems [28]. There is however a property which distinguishes innovation systems from the generic social system: that is, the capacity of the former to act as problem-solving environments [11]. An innovation system possesses the peculiar function of developing knowledge, [29] which is not necessarily possessed by the general social system [30]. It has been theorised that developing and distributing knowledge [31] is the method by which the innovation system implements the function of solving problems, [32, 33] and we will 
be working within this theoretical assumption. The innovation system, for this paper, is therefore framed as a thermodynamically-open social system which solves problems through the development and diffusion of knowledge.

Evolution and Self-organisation. Like all other social systems, [34] an innovation system undertakes evolution [35] and changes in complexity over time [36]. The change in complexity of a system, in absence of any central planning or authority, is called in the literature self-organisation [37]. Self-organisation in a system implies that the system's components do not have access to global information, but only to information which is available in their immediate neighbourhood, and that upon that information they then act [28].

Innovation systems evolve, with a process that may concern either their members, [38] their relationships and interactions, [39] the technological channels of communication, [40] the policies pursued in them, [41] or all of these factors simultaneously [42]. For the purpose of this work we will limit ourselves to consider as evolution of an innovation system the modification of the existing relationships between its members, and the functions which they perform in their system. This process of evolution of the innovation system is characterised by self-organisation, [43] and it occurs along the lines of both information [44] and knowledge flows within the system [45]. The selforganisation of an innovation system is also the result of evolutionary pressures, [46] and we will here argue that one form of such pressures is cognitive distance between organisations within a network of innovation, whose attempt at reduction may lead to modifications in the relationships within the system and to the emergence of complex structures. While it has also been suggested that variations in the complexity of an innovation system might be the consequence of intrinsic complexity of the problems to be solved, [47] it has also been suggested that problems related to the transfer of knowledge within the elements of the system can, by themselves, generate the emergence of complex network structures, through a process which is thermodynamically advantageous.

Dissipative Innovation Systems. As the innovation system acquires a more complex structure, its entropy decreases. If one assumes that the decrease in entropy follows the expenditure of some kind of energy by the system, without which its evolution towards a lower-entropy state is not possible, then it follows that the innovation system can be framed as a dissipative system. This is a consequence of the theory which, in more general terms, suggests that all social systems can be considered as dissipative systems; [48] and, among them, innovation systems can thus also be considered as dissipative systems [49].

The application of the theory of dissipative structures [50] to the study of social systems has already been done in the past, $[51,52]$ and it has also been applied to the study of innovation systems specifically, $[53,54]$ to understand the process by which new structures evolve in old organisational networks [55].

By framing the problem in this manner the emergence of a hierarchical structure in a dissipative innovation system can be considered as a process through which the innovation system reaches a different level of entropy in its structure, [56] by means of a series of steps which imply sequential minimal variations in the level of entropy of the system, and lead to the emergence of complexity [57]. 
Cognitive Distance as Noise. The process of transferring knowledge between organisations presumes the existence of knowledge assets that are transferred [58]. Companies embedded in an innovation system are therefore characterised by an intellectual or knowledge capital, [59] which is the sum of the knowledge assets which they possess, [60] and which in turn are the result of the individual organisation's path of development, [61] and of the knowledge possessed by the human and technological components of the organisation [62]. Any two organisations do not generally share the same intellectual capital, and therefore there are differences in the knowledge assets which they possess, and in the understanding and representation which they create about the world. This difference is called "cognitive distance" in the literature on knowledge management, and it refers to the difficulty in transferring knowledge between any two organisations [63].

The theory suggests that an innovation network has to perform a trade-off between increasing cognitive distance between organisations, which means higher novelty value, and increasing mutual understanding between them, which gives higher transfer of knowledge at the expenses of novelty [64]. It has been argued that if alliances (that is, network connections) are successfully formed between organisations with high cognitive distance between their members, this leads to a higher production of innovation by that alliance, [65] as a consequence of the relationship between cognitive distance and novelty, as described above. It has also been argued that the measure of centrality of a organisation in an innovation network is a consequence of the organisation's impact on the whole knowledge governance process, with organisations contributing more to it located more centrally in the network [66]. We propose that this known mechanism might play a role in the dynamic evolution of an innovation system, in a manner analogous to that of noise in an information system. The idea is that an organisation generally possessing a lower cognitive distance between multiple components of a network might spontaneously become a preferential intermediary for the transfer of knowledge within the innovation system, and as a consequence of this a hierarchical network structure emerges out of a lower-ordered structure.

\section{The Structure of the Network and Its Evolution}

\subsection{The Structure of the Network}

The modeling of the process of evolution of a network of innovation is conducted as follows. First, we imagine that there are two different structures of the ego-network of an innovation seeker company that are the subject of our analysis. The first is a horizontal network, in which a seeker organisation is positioned in a network of solvers, which are all directly connected with the seeker organisation in question. The second is a hierarchical or fractal network, in which a structure exists that represents the presence of intermediaries in the transfer of knowledge between the seeker organisation and the solving organisations in the same network.

All nodes besides the seeker organisation being studied in the first scenario, and all nodes at the periphery of the hierarchical structure of the second scenario, are from here on called solvers. There are $N$ nodes in the ego-network of an innovation seeker 
company. The $N$ nodes in the horizontal network are all solver nodes, while the $N$ nodes in the hierarchical network are divided into two classes of nodes: the intermediaries comprised of $M$ nodes, and the solvers, comprised of $M^{2}$ nodes (Fig. 1).
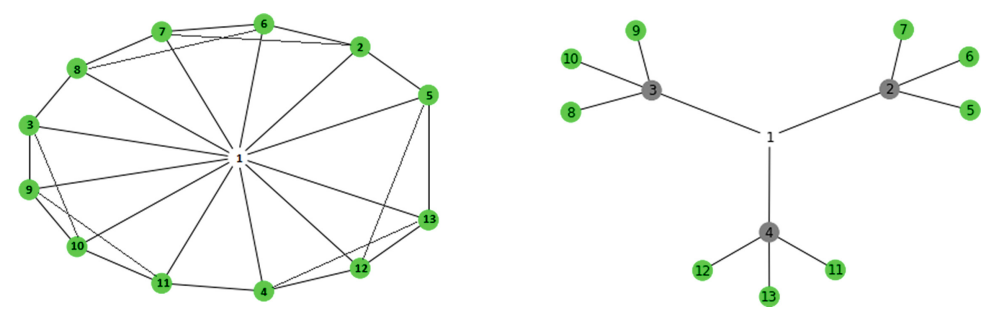

Fig. 1. In a horizontal network (to the left), all nodes in the ego-network of a seeker organisation are solver nodes (in green). In a hierarchical network (to the right), all nodes in the ego-network are either solver nodes (in green), or intermediaries (in grey). (Color figure online)

In order to make the two network structures comparable we impose the additional condition that the total number of nodes in the two networks is the same, which is satisfied for $N=M^{2}+M$. We also impose the additional condition that each of the $N$ solver nodes in the periphery of the horizontal network has at least $M$ link neighbours belonging to $N$, as this allows us to describe a dynamical process which leads from the horizontal network to the hierarchical network without the creation of new links.

\subsection{The Entropy of the Network}

The hierarchical network always possesses a lower entropy than the horizontal network comprised of the same number of nodes. This can be demonstrated by using as a measure of entropy Shannon's definition, [67] which calculates it as the amount of information required to describe the current status of a system, accordingly to the formula below:

$$
H(X)=-\sum_{i=1}^{n} p\left(x_{i}\right) \log _{2} p\left(x_{i}\right)
$$

This measure of entropy can be applied to a social network by assigning the random variable $X$ to the flattened adjacency matrix of the edges of the network, as done by others [68]. The adjacency matrices of the two classes of networks in relation to the size $N+1$ of the same network are indicated in the tables below, for the specific values $M=2 \rightarrow N=6$ (Table 1 ). 
Table 1. Adjacency matrices of two network structures for $M=2$.

\begin{tabular}{cccccccc|} 
& \multicolumn{8}{c}{ Horizontal Network } \\
\cline { 2 - 8 } node & 1 & 2 & 3 & 4 & 5 & 6 & 7 \\
\cline { 2 - 8 } 1 & 0 & 1 & 1 & 1 & 1 & 1 & 1 \\
2 & 1 & 0 & 0 & 1 & 1 & 0 & 0 \\
3 & 1 & 0 & 0 & 0 & 0 & 1 & 1 \\
4 & 1 & 1 & 0 & 0 & 0 & 0 & 1 \\
5 & 1 & 1 & 0 & 0 & 0 & 1 & 0 \\
6 & 1 & 0 & 1 & 0 & 1 & 0 & 0 \\
7 & 1 & 0 & 1 & 1 & 0 & 0 & 0 \\
\cline { 2 - 7 } & & & & & &
\end{tabular}

\begin{tabular}{|c|c|c|c|c|c|c|c|}
\hline \multicolumn{8}{|c|}{ Hierarchical Network } \\
\hline de & 1 & 2 & 3 & 4 & 5 & 6 & 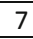 \\
\hline 1 & 0 & 1 & & 0 & J & 0 & \\
\hline 2 & & 0 & U & 1 & 1 & 0 & \\
\hline & & 0 & J & 0 & J & 1 & \\
\hline & & 1 & & 0 & & 0 & \\
\hline & & 1 & & 0 & & 0 & \\
\hline 6 & 0 & 0 & & 0 & 0 & 0 & \\
\hline & & & & & & & \\
\hline
\end{tabular}

In general, for any value $M \geq 2$, if a horizontal network has $N$ solver nodes, one seeker node is connected to all other $N$ nodes, and all solver nodes are additionally connected to $M$ solver nodes each, where $M+M^{2}=N$. In a hierarchical network with $M$ intermediary nodes and $M^{2}$ solver nodes, the seeker node is connected to $M$ intermediary nodes, and each of the intermediary nodes is connected to $M$ solver nodes. The general formulation of the adjacency matrix is indicated below, in relation to the value of $M$ (Table 2).

Table 2. General structure of the adjacency matrices

\begin{tabular}{l|l|l|l}
\hline \multicolumn{2}{l|}{ Horizontal network } & \multicolumn{2}{l}{ Hierarchical network } \\
\hline Potential links & Existing links & Potential links & Existing links \\
\hline$(N+1)^{2}$ & $2 N+N M$ & $(N+1)^{2}$ & $2 M+2 M^{2}$ \\
\hline
\end{tabular}

The adjacency matrices can be flattened by either chaining all rows or all columns together, in order to obtain a vector $X$ which univocally corresponds to a given matrix. This vector has a dimensionality of $(N+1)^{2}$, having been derived from an $N+1$ by $N+1$ matrix. The vector $X$ which derives from flattening can then be treated as the probability distribution over a random binary variable, and Shannon's measure of entropy can be computed on it. For the horizontal network, the vector $X_{\text {horizontal }}$ has value 1 two times for each of the peripheral nodes because of their connection to the centre, and then again twice for each of the peripheral nodes. This means that the vector $X_{\text {horizontal }}$ corresponds to the probability distribution (2).

$$
X_{\text {horizontal }}=\left\{\begin{array}{c}
p\left(x_{1}\right)=\frac{2 N+N M}{(N+1)^{2}}=\frac{\left(M+M^{2}\right)(M+2)}{\left(M+M^{2}+1\right)^{2}} ; \\
p\left(x_{0}\right)=1-p\left(x_{1}\right)
\end{array}\right\}
$$


For the hierarchical network, the vector $X_{\text {hierarchical }}$ has value 1 two times for each of the $M$ intermediary nodes, and then 2 times for each of the $M^{2}$ nodes. The probability distribution associated with the vector $X_{\text {hierarchical }}$ is therefore (3)

$$
X_{\text {hierarchical }}=\left\{\begin{array}{c}
p\left(x_{1}\right)=\frac{2 M+2 M^{2}}{\left(M+M^{2}+1\right)^{2}} ; \\
p\left(x_{0}\right)=1-p\left(x_{1}\right)
\end{array}\right\}
$$

The hierarchical network systematically possesses a lower level of entropy than a horizontal network with the same number of nodes, as shown in the graph below (Fig. 2).

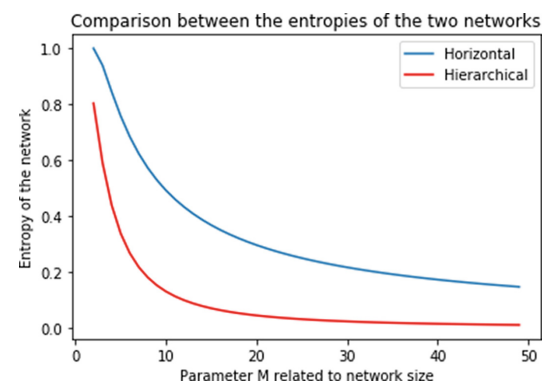

Fig. 2. Comparison between the levels of entropy of the two types of network structures.

Since we consider the network as a dissipative system, the lower level of entropy implies an expected higher energetic cost of maintenance for the lower-entropy structure. It follows from this theoretical premise that the hierarchical network should either allow the system to receive a higher input, or emit a lower output, or both simultaneously, lest its structure would decay to a higher entropy form, the horizontal one.

\subsection{The Evolutionary Dynamics of the Network}

An innovation system which starts evolving from a horizontal structure would tend to develop a hierarchical structure as a solution to the problem of transfer of knowledge in a network where cognitive distance is not uniformly distributed, as we will see in this paragraph. This can be shown by considering the hierarchical network as an attractor for the dynamical evolution of a horizontal network, under condition that the cognitive distance between pairs of nodes is distributed non-uniformly.

Stationary States. For the context of this paper, as we model a finite-state network which operates on discrete time, which models the dynamics of a dissipation systems 
which evolves over time [69]. These functions have the form depicted below, with $x(k)$ being the state of the system at time $k, u(k)$ being the input to the system at $k$, and $y(k)$ being the output of the system.

$$
\begin{gathered}
x(k+1)=f(x(k), u(k)) \\
y(k)=h(x(k), u(k))
\end{gathered}
$$

If the system does not undertake change in its internal structure, having already reached a stationary state, then $x(k+1)=x(k)$. As we want to study whether the system spontaneously evolves from a horizontal to a hierarchical structure, we can assume that $x(k+1)=f_{\text {hiearchical }}(x(k), u(k))=x(k)$ which can only be true if either the input $u(k)$ is 0 , which is not the case if the system is active, or if $u(k+1)=u(k)$. For the innovation system this condition is valid if minor variations in the structure of the network associated with it do not lead to a significant variation of the input to the system, which means that no advantages in the receipt by the seeker of solutions found by the solver should be found. If this is true, and if the hierarchical structure is an attractor for the corresponding horizontal network, then we expect the input of the horizontal network to increase as it acquires a modular structure and develops into a hierarchical network.

Input of the System. The input function of the system depends on the receipt by the seeker organisation of a solution to a problem found by one of the peripheral solver organisations, as described above. Let us imagine that at each timestep the solver organisations do indeed find a solution, and that thus the input $u(k)$ depends on the number of solver nodes, and for each of them on the probability of correct transmission of knowledge from them to the seeker organisation, which increases as the cognitive distance between two communicating nodes decreases. If this is true, then the input to the horizontal network is a function of the form $u_{\text {horizontal }}\left(N_{k}, p_{k}\right)$, where $N$ is the number of solver nodes, and $p$ is the cognitive distance in the knowledge transmission channel. Similarly, the input to the hierarchical network is a function of the form $u_{\text {hierarchical }}\left(M_{k}^{2}, q_{k}\right)$ which depends on the $M^{2}$ solver nodes in the hierarchical network, and on the parameter $q$ which describes the cognitive distance. $N$ and $M$ are such that as they increase so do, respectively, $u_{\text {horizontal }}$ and $u_{\text {hierarchical }}$; while $p$ and $q$ are such that, as they decrease, so do respectively $u_{\text {horizontal }}$ and $u_{\text {hierarchical }}$ increase. It can be also noted that $M^{2}<N \rightarrow \forall(M, N) \in \mathbb{N}$, under condition $N=M^{2}+M$ defined above. It can then be argued that if $p \leq q$ then $u_{\text {horizontal }}>u_{\text {hiearchical }}$, which means that the system would not evolve into a hierarchical network. It can also be noted that, if $N$ and $M^{2}$ are sufficiently large, then $\lim _{N, M \rightarrow+\infty}\left(N / M^{2}\right)=1$ and therefore any difference between the number of solvers in the two network structures would not play a role in the input to the innovation system. From this follows that $u_{\text {hiearchical }}>u_{\text {horizontal }} \rightarrow q<p$; that is, that the input to the innovation system with a hierarchical structure is higher than the input to the innovation system with a horizontal structure, if the cognitive distance between the members of the former is lower than the cognitive distance between the members of the latter. 
Output of the System. As per the output of the system, we can imagine that there is a cost to be paid for the maintenance of the communication channels from which the seeker receives solutions from the solvers. If the system is in a stationary state, the condition $y(k+1)=y(k)$ must be valid, as it follows from the considerations that $u(k+1)=u(k)$. If the system is not in a stationary state, as the input to the system increases, so should the output, under the hypothesis of dissipative system described above. A graphical representation of the evolution of the system from higher to lower entropy state is thus presented below (Fig. 3).

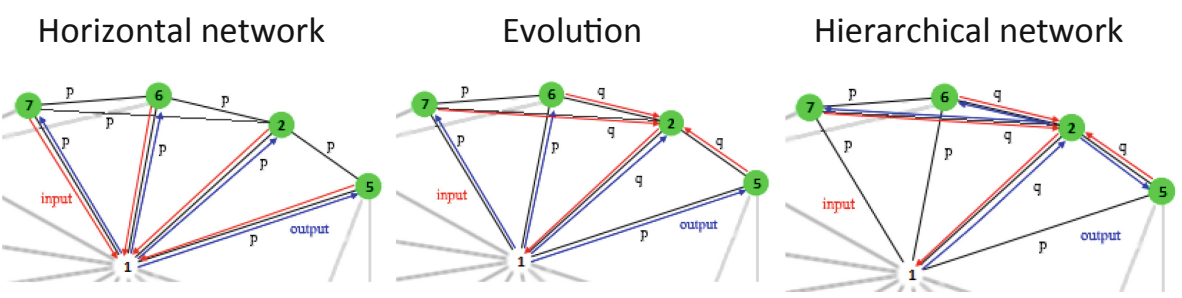

Fig. 3. Evolution of a branch of the innovation network from a higher to a lower entropy structure, from left to right. The letters $p$ and $q$ define respectively a high and a low cognitive distance between peers.

The seeker organisation would at each step receive a solution transferred by one of its link neighbours, with the indication of the full path through which the communication has reached it. The seeker would then pay a certain cost, an output with the terminology of dissipative systems, for the maintenance of the channel through which the solution has been transferred to it successfully. Such channels increase in intensity or weight, and are more likely to be used in subsequent iterations. On the contrary, channels through which a solution has not been received in a given iteration are decreased in intensity or weight, and are less likely to be used in the future. A process such as the one described would eventually, if enough iterations are performed, lead to the withering links between nodes with higher cognitive distance, and to the preservation of links between nodes with a lower cognitive distance. New connections are not formed, because cognitive distance is considered to be an exogenous parameter in this model, which does not vary once the innovation system starts evolving. Real-world phenomena are not characterised by this restriction, which should be considered when analysing real-world systems under this model.

\section{Conclusions and Future Work}

The originality of this paper consists in the framing of an innovation system under different theoretical approaches, such as that of thermodynamically-open systems, selforganisation and evolution, dissipative systems, and cognitive distance, which, when combined, highlight another way of understanding the overall operation and the evolution of innovation systems. From this perspective, the process which we here describe 
accounts for an emergent complexity of the innovation system, which can occur without central planning and on the basis of information locally available by its members. This seems to confirm the theory according to which innovation systems can self-organise to solve, among others, the problem of transfer of knowledge among their members. This seems also to suggest that, if the only form of proximity which matters is cognitive, and not geographical, organisational, or other, it might be possible to infer cognitive distance between the members of an innovation system on the basis of the way in which their relationships change over time. The theoretical prediction which this model allows to make is that, should a connection between members of an innovation system be preserved while others are dropped, this means that the cognitive distance between pairs of nodes with surviving connections is lower than that of other nodes in their ego-networks. The modelling of the evolution of an innovation system that we propose also shows that, if an innovation system starts its evolution with a centrally, highly-connected organisation in a largely horizontal network of solver, where the cognitive distance between each pair of nodes is not uniformly distributed, then the system would evolve towards a lower-entropy hierarchical structure, in order to solve the problem of transfer of knowledge from the organisations at the periphery of the innovation system to the central organisation. Our finding is consistent with the theory on modularity as an emergent property of complex adaptive innovation systems. Subsequent research might apply the mathematical model described in this paper to a longitudinal study of the evolution of real-world innovation networks, in order to test whether the theory related to the spontaneous emergence of a hierarchical structure of innovation networks can be empirically supported. On the theoretical plane, further research could expand the understanding of the evolution of an innovation network by adding considerations related to the role which geographical and organisational proximity have in the development of the network, and add these factors to the model proposed. Issues related to perturbation of the network, limit cycle of its evolution, and self-organised criticality in connection to our model may also be explored in subsequent works.

\section{References}

1. Simmel, G.: Sociologie et épistémologie, Paris, PUF, collection «Sociologie», 1981 (1917)

2. Taalbi, J.: Evolution and structure of technological systems - an innovation output network (2018). https://arxiv.org/pdf/1811.06772.pdf

3. Russell, M.G., Smorodinskaya, N.V.: Leveraging complexity for ecosystemic innovation. Technol. Forecast. Soc. Chang. 136, 114-131 (2018)

4. Jackson, D.J.: What is an innovation ecosystem. National Science Foundation, 1 (2011)

5. Parker, L.A.: Networks for innovation and problem solving and their use for improving education: a comparative overview (1977)

6. Lubik, S., Garnsey, E., Minshall, T., Platts, K.: Value creation from the innovation environment: partnership strategies in university spin-outs. R\&D Management 43(2), 136150 (2013)

7. Burt, R.S.: Structural Holes: The Social Structure of Competition. Harvard university press, Cambridge (2009) 
8. Roos, G.: Knowledge management, intellectual capital, structural holes, economic complexity and national prosperity. J. Intell. Capital 18(4), 745-770 (2017)

9. Leydesdorff, L., Rotolo, D., de Nooy, W.: Innovation as a nonlinear process, the scientometric perspective, and the specification of an 'innovation opportunities explorer'. Technol. Anal. Strateg. Manag. 25(6), 641-653 (2013)

10. Sáenz-Royo, C., Gracia-Lázaro, C., Moreno, Y.: The role of the organization structure in the diffusion of innovations. PLoS ONE 10(5), e0126076 (2015)

11. Terwiesch, C., Xu, Y.: Innovation contests, open innovation, and multiagent problem solving. Manage. Sci. 54(9), 1529-1543 (2008). https://doi.org/10.1287/mnsc.1080.0884

12. Cowan, R., Jonard, N.: Network structure and the diffusion of knowledge. J. Econ. Dyn. Control 28(8), 1557-1575 (2004)

13. Brusoni, S.: The limits to specialization: problem solving and coordination in "modular networks". Organ. Stud. 26(12), 1885-1907 (2005). https://doi.org/10.1177/ 0170840605059161

14. Ravasz, E., Barabási, A.-L.: Hierarchical organization in complex networks. Phys. Rev. E, 67(2) (2003) https://doi.org/10.1103/physreve.67.026112

15. Barabási, A.L., Dezső, Z., Ravasz, E., Yook, S.H., Oltvai, Z.: Scale-free and hierarchical structures in complex networks. In: AIP Conference Proceedings, vol. 661, no. 1, pp. 1-16. AIP, April 2003

16. Katz, J.S.: What is a complex innovation system? PLoS ONE 11(6), e0156150 (2016)

17. Katz, J.S., Ronda-Pupo, G.A.: Cooperation, scale-invariance and complex innovation systems: a generalization. Scientometrics (2019). https://doi.org/10.1007/s11192-01903215-8

18. Zhang, J.: Growing Silicon Valley on a landscape: an agent-based approach to high-tech industrial clusters. In: Industry And Labor Dynamics: The Agent-Based Computational Economics Approach, pp. 259-283 (2004)

19. Spielman, D.J., Ekboir, J., Davis, K.: Developing the art and science of innovation systems enquiry: alternative tools and methods, and applications to sub-Saharan African agriculture. In: Innovation Africa, pp. 98-112. Routledge (2012)

20. Brent, S.B.: Prigogine's model for self-organization in nonequilibrium systems. Hum. Dev. 21(5-6), 374-387 (1978)

21. Harvey, D.L., Reed, M.H.: The evolution of dissipative social systems. J. Soc. Evol. Syst. 17 (4), 371-411 (1994)

22. Chick, V., Dow, S.: The meaning of open systems. J. Econ. Methodol. 12(3), 363-381 (2005)

23. Luhmann, N.: Social Systems. Stanford University Press, Stanford (1995)

24. Fischer, M.M., Fröhlich, J. (Eds.) Knowledge, Complexity and Innovation Systems. Advances in Spatial Science (2001). https://doi.org/10.1007/978-3-662-04546-6

25. Amable, B.: Institutional complementarity and diversity of social systems of innovation and production. Rev. Int. Polit. Econ. 7(4), 645-687 (2000)

26. Gómez-Uranga, M., Etxebarria, G.: Thermodynamic properties in the evolution of firms and innovation systems (2015). SSRN 2697747

27. Saviotti, P.P.: Networks, national innovation systems and self-organisation. In: Fischer, M. M., Fröhlich, J. (eds.) Knowledge, Complexity and Innovation Systems. Advances in Spatial Science, pp. 21-45. Springer, Heidelberg (2001). https://doi.org/10.1007/978-3-662-045466_2

28. Hemelrijk, C. (ed.): Self-organisation and Evolution of Biological and Social Systems. Cambridge University Press, Cambridge (2005) 
29. Hekkert, M.P., Suurs, R.A.A., Negro, S.O., Kuhlmann, S., Smits, R.E.H.M.: Functions of innovation systems: a new approach for analysing technological change. Technol. Forecast. Soc. Chang. 74(4), 413-432 (2007). https://doi.org/10.1016/j.techfore.2006.03.002

30. Collins, R.: On the sociology of intellectual stagnation: the late twentieth century in perspective. Theory Cult. Soc. 9(1), 73-96 (1992)

31. Yan, E.: Disciplinary knowledge production and diffusion in science. J. Assoc. Inf. Sci. Technol. 67(9), 2223-2245 (2016)

32. Nickerson, J.A., Zenger, T.R.: A knowledge-based theory of the organisation-the problemsolving perspective. Organ. Sci. 15(6), 617-632 (2004)

33. Eisner, H.: Thinking: A Guide to Systems Engineering Problem-solving. CRC Press, Boca Raton (2019)

34. Kappeler, P.M., Clutton-Brock, T., Shultz, S., Lukas, D.: Social complexity: patterns, processes, and evolution (2019)

35. Pyka, A., Foster, J. (eds.): The Evolution of Economic and Innovation Systems. ECE. Springer, Cham (2015). https://doi.org/10.1007/978-3-319-13299-0

36. Frenken, K.: A complexity-theoretic perspective on innovation policy. Complex. Innov. Policy 3(1), 35-47 (2017)

37. De Wolf, T., Holvoet, T.: Emergence versus self-organisation: different concepts but promising when combined. In: Brueckner, Sven A., Di Marzo Serugendo, G., Karageorgos, A., Nagpal, R. (eds.) ESOA 2004. LNCS (LNAI), vol. 3464, pp. 1-15. Springer, Heidelberg (2005). https://doi.org/10.1007/11494676_1

38. Kastelle, T., Potts, J., Dodgson, M.: The evolution of innovation systems. In: DRUID Summer Conference, June 2009

39. Feldman, M.P., Feller, I., Bercovitz, J.L., Burton, R.: Understanding evolving universityindustry relationships. In: Feldman, M.P., Link, A.N. (eds.) Innovation policy in the knowledge-based economy. Economics of Science, Technology and Innovation, 23rd edn, pp. 171-188. Springer, Boston (2001). https://doi.org/10.1007/978-1-4615-1689-7_8

40. Murray, F.: Innovation as co-evolution of scientific and technological networks: exploring tissue engineering. Res. Policy 31(8-9), 1389-1403 (2002)

41. Cooke, P.: From technopoles to regional innovation systems: the evolution of localised technology development policy. Can. J. Region. Sci. 24(1), 21-40 (2001)

42. Trippl, M., Grillitsch, M., Isaksen, A., Sinozic, T.: Perspectives on cluster evolution: critical review and future research issues. Eur. Plan. Stud. 23(10), 2028-2044 (2015)

43. Silverberg, G., Dosi, G., Orsenigo, L.: Innovation, diversity and diffusion: a selforganisation model. Econ. J. 98(393), 1032-1054 (1988)

44. Fuchs, C. (2002). Social information and self-organisation

45. Fuchs, C., Hofkirchner, W.: Self-organization, knowledge and responsibility. Kybernetes 34 $(1 / 2), 241-260(2005)$

46. Etzkowitz, H., Leydesdorff, L.: The dynamics of innovation: from National Systems and "Mode 2" to a Triple Helix of university-industry-government relations. Res. Policy 29(2), 109-123 (2000). https://doi.org/10.1016/s0048-7333(99)00055-4

47. Brusoni, S., Marengo, L., Prencipe, A., Valente, M.: The value and costs of modularity: a problem-solving perspective. Eur. Manag. Rev. 4(2), 121-132 (2007). https://doi.org/10. 1057/palgrave.emr.1500079

48. Xiong, F., Liu, Y., Zhu, J., Zhang, Z.J., Zhang, Y.C., Zhang, Y.: A dissipative network model with neighboring activation. Eur. Phys. J. B 84(1), 115-120 (2011). https://doi.org/ 10.1140/epjb/e2011-20286-7

49. Diao, Z.F., Zhang, F.S.: The Analysis of dissipative structure in the technological innovation system of enterprises. J. Jiangnan Univ. (Human. Soc. Sci. Edn.) 2 (2009) 
50. Prigogine, I., Kondepudi, D.: Modern Thermodynamics: From Heat Engines to Dissipative Structures, 2nd edn. Wiley, New York (1998)

51. Kiel, L.D.: Lessons from the nonlinear paradigm: applications of the theory of dissipative structures in the social sciences. Soc. Sci. Q. (1991)

52. Schieve, W.C., Allen, P.M. (eds.): Self-organization and Dissipative Structures: Applications in the Physical and Social Sciences. University of Texas Press, Austin (1982)

53. Leifer, R.: Understanding organizational transformation using a dissipative structure model. Hum. Relat. 42(10), 899-916 (1989)

54. Jenner, R.A.: Technological paradigms, innovative behavior and the formation of dissipative enterprises. Small Bus. Econ. 3(4), 297-305 (1991)

55. Gemmill, G., Smith, C.: A dissipative structure model of organization transformation. Hum. Relat. 38(8), 751-766 (1985). https://doi.org/10.1177/001872678503800804

56. Li, Z., Jiang, J.: Entropy model of dissipative structure on corporate social responsibility. In: IOP Conference Series: Earth and Environmental Science, vol. 69, no. 1, p. 012126). IOP Publishing, June 2017

57. Basile, G., Kaufmann, H.R., Savastano, M.: Revisiting complexity theory to achieve strategic intelligence. Int. J. Foresight Innov. Policy 13(1-2), 57-70 (2018)

58. Girard, J., Girard, J.: Defining knowledge management: toward an applied compendium. Online J. Appl. Knowl. Manag. 3(1), 1-20 (2015)

59. Laperche, B.: Enterprise Knowledge Capital. Wiley, Hoboken (2017)

60. Marr, B., Schiuma, G., Neely, A.: Intellectual capital-defining key performance indicators for organizational knowledge assets. Bus. Process Manag. J. 10(5), 551-569 (2004)

61. Moustaghfir, K.: The dynamics of knowledge assets and their link with firm performance. Measuring Bus. Excell. 12(2), 10-24 (2008)

62. Wu, W.W., Kan, H.L., Liu, Y.X., Kim, Y.: Management mechanisms, technological knowledge assets and firm market performance. Stud. Sci. Sci. 5, 14 (2017)

63. Nooteboom, B.: Problems and solutions in knowledge transfer (2001)

64. Wuyts, S., Colombo, M.G., Dutta, S., Nooteboom, B.: Empirical tests of optimal cognitive distance. J. Econ. Behav. Organ. 58(2), 277-302 (2005). https://doi.org/10.1016/j.jebo.2004. 03.019

65. Filiou, D., Massini, S.: Industry cognitive distance in alliances and firm innovation performance. R\&D Manag. 48(4), 422-437 (2018)

66. Zhao, J., Xi, X., Guo, T.: The impact of focal firm's centrality and knowledge governance on innovation performance. Knowl. Manag. Res. Pract. 16(2), 196-207 (2018). https://doi.org/ $10.1080 / 14778238.2018 .1457004$

67. Shannon, C.E., Weaver, W.: The mathematical theory of communication. University of Illinois Press, Urbana (1949)

68. De Domenico, M., Granell, C., Porter, M.A., Arenas, A.: The physics of spreading processes in multilayer networks. Nat. Phys. 12(10), 901-906 (2016)

69. Tan, Z., Soh, Y.C., Xie, L.: Dissipative control for linear discrete-time systems. Automatica 35(9), 1557-1564 (1999). https://doi.org/10.1016/s0005-1098(99)00069-2 